\title{
Photo- and cathodoluminescence spectra of diamond single crystals formed by sintering of detonation nanodiamond
}

\author{
S. V. Kidalov ${ }^{1}$, M. V. Zamoryanskaya ${ }^{1}$, V. A. Kravez ${ }^{1}$, L. V. Sharonova ${ }^{1}$, F. M. Shakhov ${ }^{1}$, E. B. Yudina ${ }^{1}$, \\ T. O. Artamonova ${ }^{2}$, M. A. Khodorkovskii ${ }^{2}$, A. Ya. Vul ${ }^{1}$ \\ ${ }^{1}$ Ioffe Institute, Polytechnicheskaya 26, St. Petersburg, 194021, Russia \\ ${ }^{2}$ Peter the Great St. Petersburg Polytechnic University, Polytechnicheskaya 29, St. Petersburg, 195251, Russia \\ alexandervul@mail.ioffe.ru
}

\begin{abstract}
The photo- and cathodoluminescence spectra (PL, CL) of diamond single crystals synthesized under high-pressure and at high-temperature (HPHT sintering) from detonation nanodiamonds (DND) particles with Gd and Eu ion-functionalized surfaces have been studied. The HPHT sintering was made under pressures $(P \cong 7 \mathrm{GPa})$ and temperature $\left(T=1300-1500{ }^{\circ} \mathrm{C}\right)$. The DND particles have sizes of $4-5 \mathrm{~nm}$, hydrocarbons and/or alcohols were used at the HPHT synthesis instead of traditional metal catalysts. The initial and synthesized crystals were characterized by X-ray microanalysis, mass-spectroscopy, scanning electron microscopy (SEM), PL and CL. Mass spectra confirmed that Gd and $\mathrm{Eu}$ atoms were inserted into the volume of diamond single crystals in the HPHT sintering. A significant conversion of the PL and CL spectra of synthesized diamond single crystals compared with HPHT diamond crystals were observed. Transformation of the point defect assemblies inherent to the diamond crystal can explain the observed spectra.
\end{abstract}

Keywords: detonation nanodiamond, surface modification, HPHT synthesis, diamond single crystal, photoluminescence of diamond, cathodoluminescence of diamond, defects in diamond, $\mathrm{N}-\mathrm{V}$ centers, $3 d-4 f$ elements.

Received: 8 December 2018

Revised: 11 January 2019

\section{Introduction}

The recent increase of interest in nanodiamonds is related to their potential utility as nanoparticles in bioimaging systems [1-3]. The idea is related to the usage of light emission from luminescent centers in diamond lattice. Currently-known centers are nitrogen-vacancy, silicon-vacancy color centers $[4,5]$ and impurity of Eu ions in diamond lattice [6,7]. Gadolinium atoms are particularly attractive as a dopant in diamond lattice due to their potential use in nuclear magnetic resonance tomography [8].

Recently, we developed a method for producing of detonation nanodiamonds (DND) hydrosol with mean size of particles $4-5 \mathrm{~nm}[9,10]$ and submitted technological protocol for surface modification of DND particles by metal ions [11]. We also demonstrated an interesting effect of single diamond crystal formation from DND particles at high-pressure and high-temperature (HPHT) sintering [12].

It is readily obvious that it would be very attractive to study the possibility of metal ion encapsulation in diamond crystalline structure using DND surface modified particles as starting materials for HPHT sintering. This paper submits the first results from these studies.

\section{Experimental}

\subsection{Samples}

We studied following three types of samples. The first one was diamond single crystals (DSC) produced by sintering of DND powder at HPHT conditions in the presence of alcohols and/or hydrocarbons in a high-pressure chamber. The procedure of DND sintering is described in detail in $[11,12]$. The second type was DSC sintered at the same conditions from DND with surface modified with europium (Eu). The third type was DSC sintered at the same conditions from DND with surface modified with gadolinium (Gd).

The initial DND, with size of $4-5 \mathrm{~nm}$, were purified and de-agglomerated in accordance with a procedure described in $[9,10]$. Surface modification of DND with $3 d-4 f$ elements (Eu, Gd) was carried out using ionic exchange in accordance to the procedure described in $[13,14]$.

The synthesized DSC samples represent a powder of diamond single crystals raging of up to $5 \mu \mathrm{m}$ (see Fig. 1). 


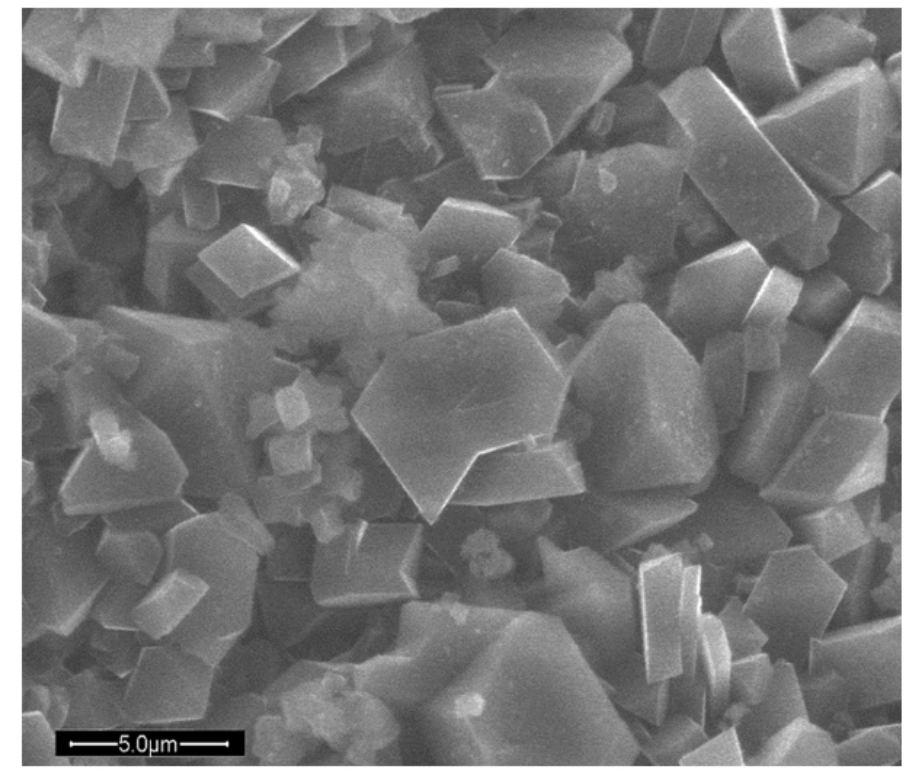

FIG. 1. SEM image of DSC obtained by HPHT sintering of DND modified with $3 d-4 f$ elements. Scale bar is 5 micron

\subsection{Methods}

The composition of the diamonds studied by energy-dispersive X-ray spectroscopy (EDX) using X-ray microanalyzer CAMEBAX-MICROBEAM from CAMECA, equipped with four X-ray spectrometers with a set of crystal analyzers. Quantitative X-ray spectral microanalysis was carried out under the following conditions: accelerating voltage - $20 \mathrm{KV}$ (electron energy $20 \mathrm{KeV}$ ), angle of X-ray selection $-40^{\circ}$, current of absorbed electrons $-10-$ $50 \mathrm{nA}$. The detection limit of the rare-earth elements in the carbon matrix was under optimal conditions in the range $0.02-0.05$ wt.\%. As the concentration approaches this value, the microanalysis became semiquantitative, the relative error could reach $50-100 \%$. To obtain the average content of $3 d-4 f$ elements, the analysis have been performed with a defocused electron probe $50 \mu \mathrm{m}$ in diameter.

Preparation of samples for mass-spectroscopy with laser evaporation consisted in gluing a portion of diamond powder to the target using a double-sided adhesive tape. Measurements performed using mass spectrometer with ion cyclotron resonance and Fourier transform (Model 902-MS MALDI Mass Spectrometer, Varian). The spectrometer was equipped with a superconducting magnet of 9.4 Tesla. Measurements were performed in the positive ion mode.

The laser evaporation of the samples performed using third harmonic of the Nd: YAG laser ( $355 \mathrm{~nm})$, whose power can be widely changed. We used DPSS (Diode-pumped solid-state) Nd:YAG laser with titanyl potassium phosphate crystal (KTiOPO4, KTP) for frequency doubling. The accuracy of the measured monoisotopic mass after calibration was better than 5 ppm.

The PL study was carried out on spectrometer Ocean Optics Maya 2000 Pro in spectral range $200-1100 \mathrm{~nm}$, using $532 \mathrm{~nm}$ laser for excitation. The resolution of the spectrometer was $0.45 \mathrm{~nm}$.

Cathodoluminescence (CL) spectra were measured on samples of diamond powder pressed into indium and carbon film with a thickness of $100 \mathrm{~nm}$ deposited on diamonds. The spectra obtained at room temperature. Energy of incident electron beam was $15 \mathrm{KeV}$ [15].

\section{Results and discussion}

According to EDX measurements, the europium and gadolinium content in DSC samples obtained by HPHT sintering of DND with modified surface was $0.01-0.02$ at.\% for both Gd and Eu samples.

Unfortunately, EDX technique is unable to distinguish $3 d-4 f$ atoms incorporated into crystal structure and deposited on the surface of diamond crystal. Thus, it is insufficient to give comprehensive evidence for presence of Gd or Eu inside DSC obtained by sintering of DND particles with modified surface.

\subsection{Mass spectroscopy}

Figure 2(a) presents mass spectrum of DSC obtained by sintering from initial DND without surface modification. 

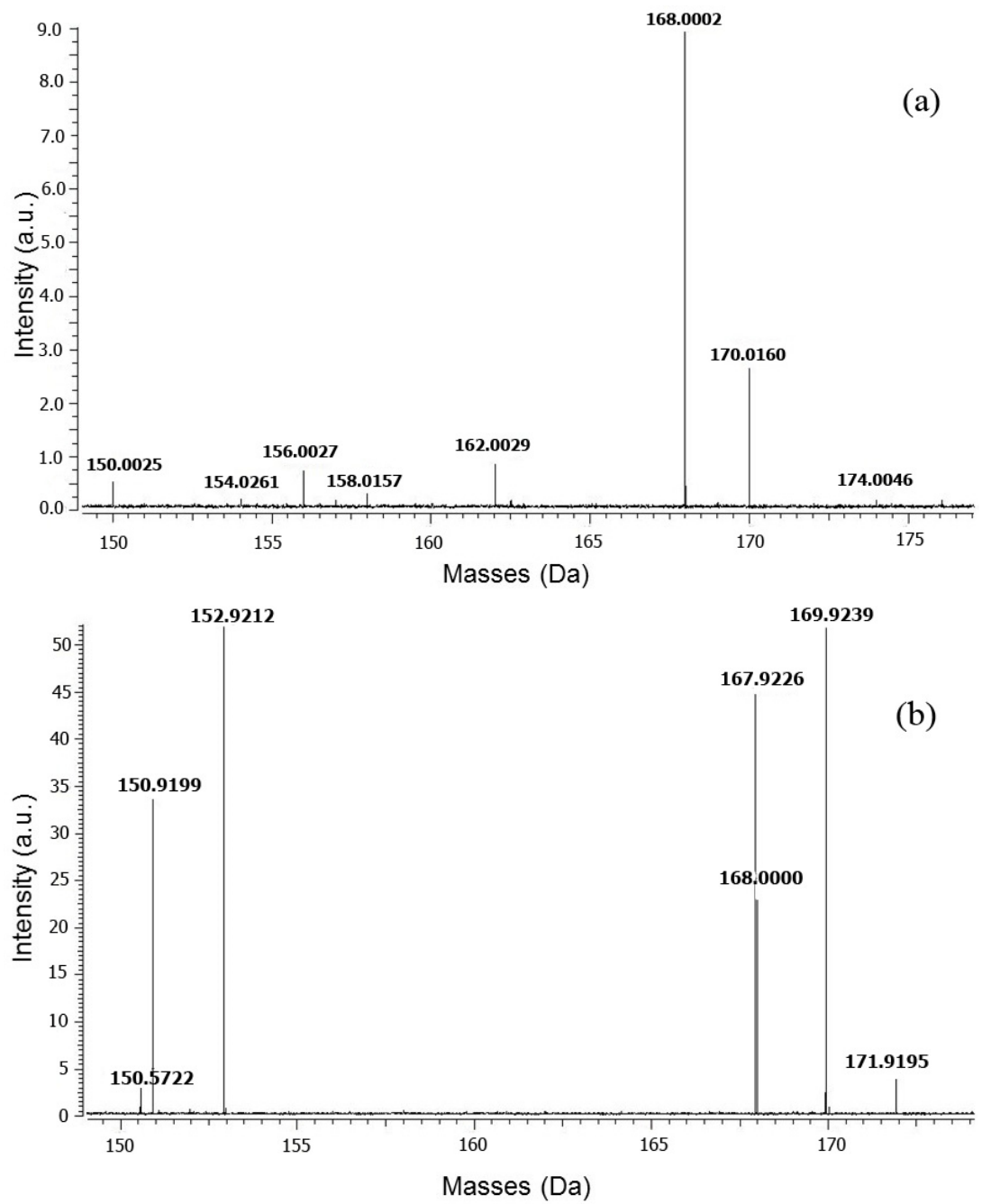

$\mathrm{Eu}(150.9198,152.9212), \mathrm{EuOH}(167.9226,169.9240)$

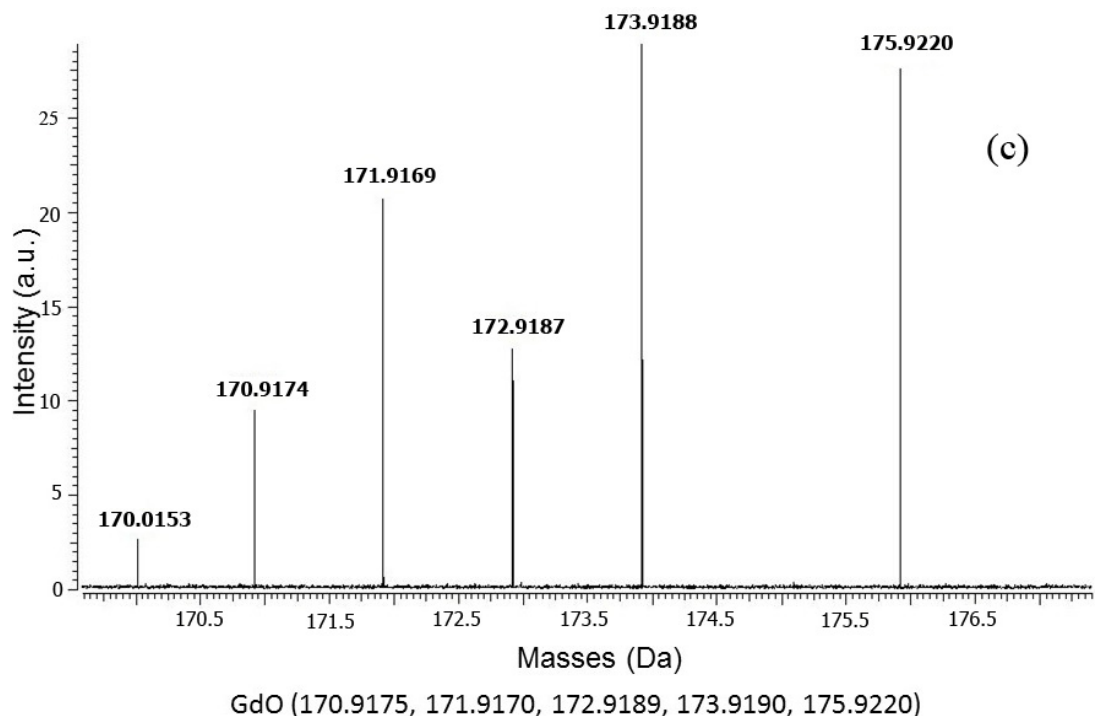

GdO $(170.9175,171.9170,172.9189,173.9190,175.9220)$

FIG. 2. Mass spectra of DSC samples obtained with use of laser evaporation: (a) from sample obtained by HPHT sintering of pure DND; (b) from sample obtained by HPHT sintering of DND with surface modified by Eu; (c) from sample obtained by HPHT sintering of DND with surface modified by Gd 
The spectrum is measured using laser evaporation of diamond crystals. In the range of $150-175$ AMU, it demonstrates a number of peaks corresponding to pure carbon atomic clusters (at 156.0027, 168.0002) and clusters containing nitrogen $(158.0157,170.0160)$, or both oxygen and nitrogen $(150.0025,162.0029,174.046,154.0261)$. The presence of oxygen and nitrogen is typical for surface of diamond crystals obtained by HPHT sintering. The same spectra demonstrate DSC samples prepared by sintering of DND modified by Eu as well as by Gd ions. At that, observed no lines that could attributed to Gd and Eu.

Figure 2(b) demonstrates mass spectrum at same band of masses of DSC sample produced by sintering DND modified by Eu. The spectrum measured at an order of magnitude increased compared to spectra in Fig. 2(a) power of evaporating laser. Along with prominent line of carbon clusters at mass 168.000, it demonstrates a series of intensive lines corresponding to europium isotopes $(150.9198,152.9212)$ and europium hydroxide EuOH (167.9226, 169.9240).

In Fig. 2(c), a mass spectrum of DSC sample produced by sintering DND modified by Gd is presented. In the band of masses 169.5 - 178.5, we observed noticeable line related to nitrogen-containing carbon clusters (170.0153). The observed series of intensive lines correspond to gadolinium oxide GdO (170.9175, 171.9170, 172.9189, 173.9190 and 175.9220). The fact that signals related to Eu and Gd appear only at increased intensity of evaporating laser irradiation suggests that corresponding atoms are situated not at the surface but inside the bulk of micron sized diamond particles.

\subsection{Cathodeluminescence spectra}

Figure 3 demonstrates the comparison of CL spectra measured on three types of samples: initial DND (curve 1), SDC sintered of initial DND (curve 2), SDC sintered of DND with surface modified by Eu ions (curve 3). Taking into account that the shape of CL spectra of diamond determined mainly by system of structural defects inside crystalline lattice of particles and contribution of surface [16], we can conclude that SDC conserve the system of defects inherited from the same of parental DND. The moderate shift to shorter wavelength range can be attributed to decrease of contribution of effective surface of diamond particles due to increase of crystalline size as a result of sintering process.

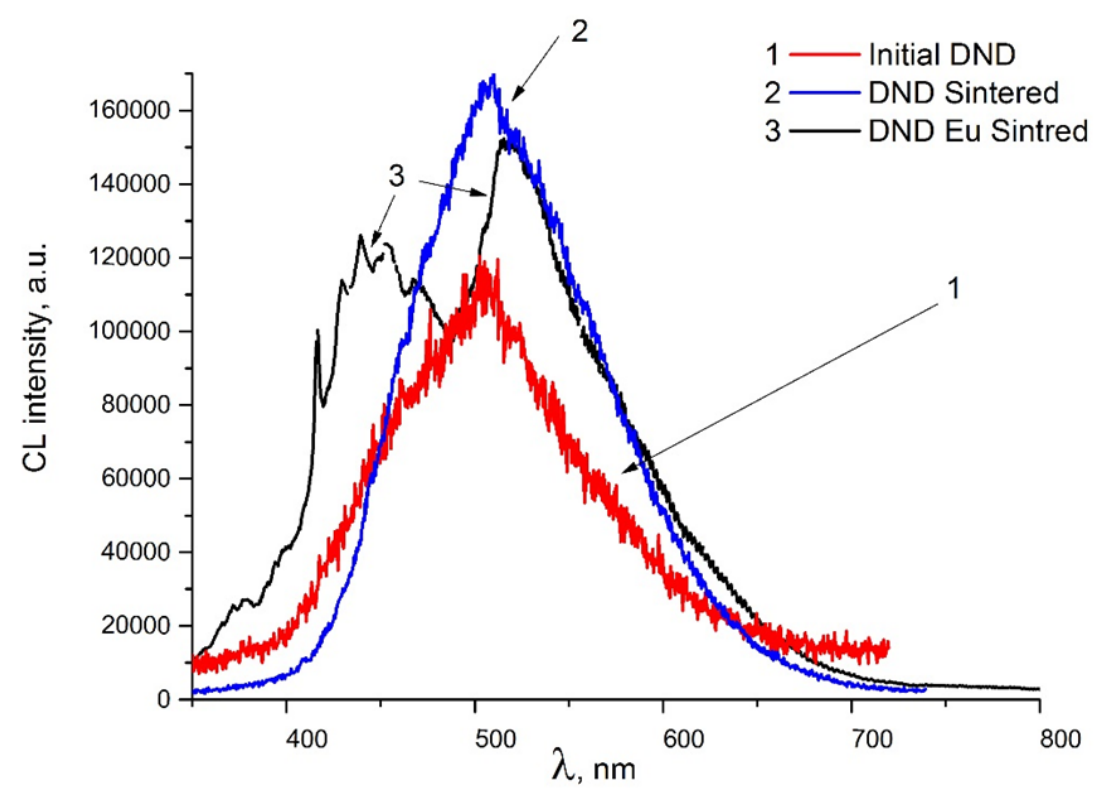

FIG. 3. Comparison of CL spectra from initial DND, DSC obtained from initial DND and DSC obtained from DND with surface modified by Eu ions

CL spectra of SDC obtained by sintering of DND with surface modified by Eu ions exhibit dramatic differences compared to the same spectra measured of SDC having no Eu. The presence of two peaks reveals significant transformation of bulk structure and increase of defects in crystalline structure of the SDC. The propagation of luminescence to short wavelength region can manifest formation the structural defects of diamond lattice obviously related to the difference between effective radius of carbon and europium atoms incorporated into diamond. Forming the breakdown in the middle of spectral range and corresponding longwave apex may be concerned to quenching the luminescence of nitrogen color centers at corresponding wavelengths due to distortion of diamond 
crystalline lattice by intrusion of $3 d-4 f$ atoms. The observed dramatic transformation of CL spectra related to Eu atoms in structure of SDC samples under study suggests that structural modification of diamond lattice is most likely due to the presence of incorporated atoms in position far from crystal surface.

\subsection{Photoluminescence spectra}

Figure 4 demonstrates photoluminescence (PL) spectra for the three types of samples. The first spectrum measured on DSC sintered of initial DND containing no $3 d-4 f$ elements (curve 1). Second spectrum was measured on DSC sintered of DND modified by Gd (curve 2). The third spectrum corresponds to DSC sintered of DND modified by $\mathrm{Eu}$ (curve 3). The spectrum of DSC containing no $3 d-4 f$ elements is typical for diamond crystals obtained by sintering of DND in the presence of hydrocarbons at high pressure and temperature [17].

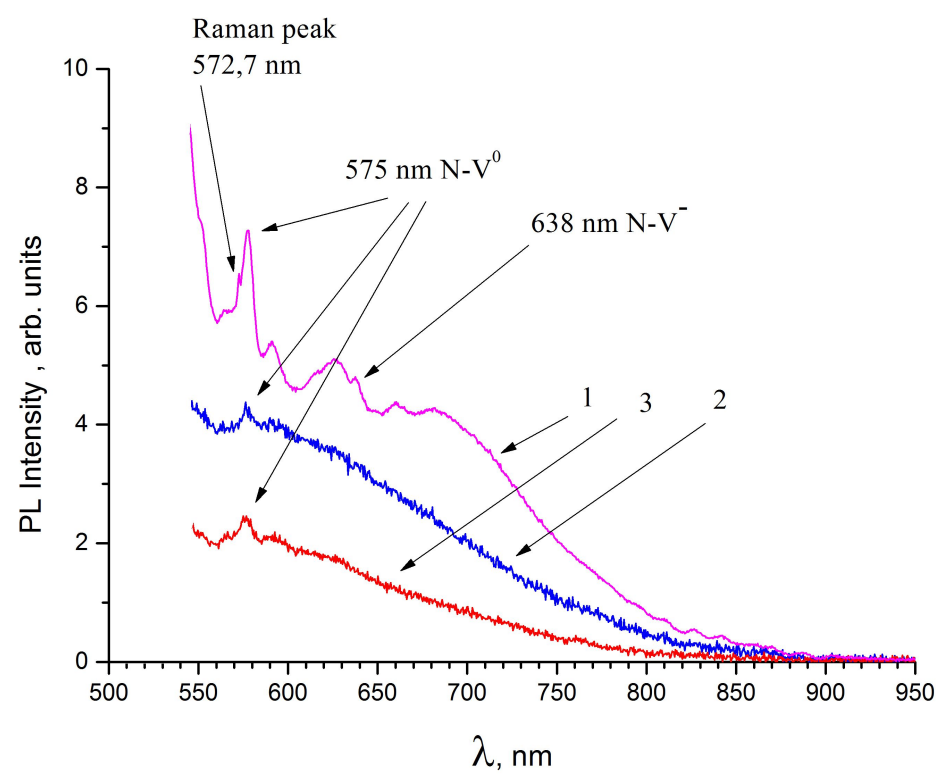

FIG. 4. PL spectra of diamond single crystals produced by HPHT sintering of DND: curve 1 - PL spectrum of diamond single crystals produced from pure DND curves 2 and 3 crystals produced from DND with surface modified by $3 d-4 f$ ions (Gd - curve 2, Eu - curve 3 )

The spectrum demonstrates clearly visible lines of nitrogen-vacancy color centers and detectable feature attributed to Raman scattering (curve 1 in Fig. 4). Photoluminescence of DSC containing Gd and Eu exhibits noticeably smaller intensity and nearly complete absence of expressed lines from nitrogen centers. Most likely, it is related to additional distortion of diamond lattice due to incorporation of large sized and heavy $3 d-4 f$ atoms. The additional vacancies and distorted bond lengths promote quenching of luminescence of color centers in diamond lattice. Thus, comparison of PL spectra from samples of DSC sintered of DND with $3 d-4 f$ atoms on surface to spectra from DSC sintered of pure DND demonstrates successful incorporation of Eu and Gd into the lattice of diamond single crystals.

\section{Conclusions}

The results of experiments support the possibility of obtaining diamond crystals containing incorporated $3 d-4 f$ elements by sintering from DND particles with surface modified by corresponding ions.

We have successfully demonstrated incorporation of $\mathrm{Eu}$ and $\mathrm{Gd}$ atoms into structure of micron-sized diamond single crystals during sintering from DND particles. Analysis of mass spectra measured with help of laser ablation confirms the presence of Eu and Gd inside diamond crystals. The comparison of CL as well as PL spectra of diamond crystals, sintered from DND with and without surface modification by Eu or Gd reveals transformation of system of defects due to the insertion of large sized $3 d-4 f$ atoms inside diamond lattice. 


\section{Acknowledgements}

The research was supported by the Russian Science Foundation (Project 14-13-00795). Mass spectrometry was carried out using scientific equipment of the Center of Shared Usage "The analytical center of nano- and biotechnologies of SPbPU”. Authors thanks to Dr. Artur Dideikin for very useful discussion and advices.

\section{References}

[1] Guodong Xia, et al. Selective phase synthesis of a high luminescence $\mathrm{Gd}_{2} \mathrm{O}_{3}$ :Eu nanocrystal phosphor through direct solution combustion. Nanotechnology, 2010, 21, 345601.

[2] Antic B., et al. Optimization of photoluminescence of $\mathrm{Y}_{2} \mathrm{O}_{3}:$ Eu and $\mathrm{Gd}_{2} \mathrm{O}_{3}$ :Eu phosphors synthesized by thermolysis of 2,4-pentanedione complexes. Nanotechnology, 2010, 21, 245702.

[3] Aharonovich I. Engineering chromium-related single photon emitters in single crystal diamonds. New Journal of Physics, 2011, 13, 045015.

[4] Shenderova O.A., et al. Nitrogen control in nanodiamond produced by detonation shock-wave-assisted synthesis. J. Phys. Chem. C, 2011, 115, P. 14014-14024.

[5] Neu E., Steinmetz D., et al. Single photon emission from silicon-vacancy colour centres in chemical vapour deposition nano-diamonds on iridium. New J. Phys., 2011, 13, 025012.

[6] Magyar A., Hu W., et al. Synthesis of luminescent europium defects in diamond. Nature Communications, $2014,5,3523$.

[7] Sedov V.S., Kuznetsov S.V., et al. Diamond-EuF3 nanocomposites with bright orange photoluminescence. Diamond and Related Materials, 2017, 72, P. 47-52.

[8] Nakamura T., Ohana T., et al. Simple Fabrication of Gd(III)-DTPA-Nanodiamond Particles by Chemical Modification for Use as Magnetic Resonance Imaging (MRI) Contrast Agent. Applied Physics Express, 2013, 6, 0150016.

[9] Aleksenskiy A.E., Eydelman E.D., Vul' A.Ya. Deagglomeration of detonation nanodiamonds. Nanosci. Nanotech. Lett., 2011,3 , P. 68-74.

[10] Dideikin A.T., Aleksenskii A.E., et al. Rehybridization of carbon on facets of detonation diamond nanocrystals and forming hydrosols of individual particles. Carbon, 2017, 122, P. 737-745.

[11] Kidalov S.V., Shakhov F.M., et al. Growth of diamond microcrystals by the oriented attachment. Tech. Phys. Lett., 2017, 43, P. 53-56.

[12] Dideikin A.T., Eidelman E.D., et al. Oriented-attachment growth of diamond single crystal from detonation nanodiamonds. Diam. Relat. Mater., 2017, 75, P. 85-90.

[13] Aleksenskii A.E., Yagovkina M.A., Vul A.Ya. Intercalation of ultrafine-dispersed diamond in aqueous suspensions. Physics of the Solid State, 2004, 46, P. 685-686.

[14] Panich A.M., Shames A.I., et al. Magnetic Resonance Study of Gadolinium-Grafted Nanodiamonds. The Journal of Physical Chemistry C, 2016, 120, P. 19804-19811.

[15] Zamoryanskaya M.V., Konnikov S.G., Zamoryanskii A.N. A high-sensitivity system for cathodoluminescent studies with the Camebax Electron Probe Microanalyzer. Instruments and Experimental Techniques, 2004, 47, P. 477-483.

[16] Zaitsev A.M. Optical properties of diamond. Berlin, Heidelberg: Springer-Verlag, 2001.

[17] Kidalov S.V., Shnitov V.V., et al. Chemical composition of surface and structure of defects in diamond single crystals produced from detonation nanodiamonds. Nanosystems: Physics, Chemistry, Mathematics, 2018, 9 (1), P. 21-24. 